\title{
El caso sede México: Formación en Educación Física en e-entornos universitarios durante la contingencia de la COVID-19 The México heartquarters case: Physical Education Teacher Education in e- university environments during the COVID-19 contingency \\ Ana M aria de Guadal upe Arras-Vota, J osé Luis Bordas-Beltrán, Fernando M ondaca-Fernández, Juan M anuel Rivera-Sosa \\ Universidad Autónoma de Chihuahua (M éxico)
}

\begin{abstract}
Resumen. El objetivo de la presente investigación fue describir los desafíos enfrentados en cuanto al uso de Educación Virtual durante la contingencia de la COVID-19, por 254 docentes U niversitarios del área de Educación Física(153 mujeres, 102 hombres) de cuatro Instituciones de Educación Superior (IES) en M éxico, todos del área de Educación Física (EF). Por medio de cuestionarios online, a través de métodos: analítico - sintético, teórico - deductivo y hermenéutico; y técnicas cuantitativas y cualitativas, se recabó lainformación que permitió concluir que los docentes en este estudio: 1. No se consideran capacitados para utilizar eficientemente la educación virtual; 2 . Consideran que la educación virtual, en EF, no ofrece los mismos beneficios que la presencial, 3. El aislamiento por lacontingencia es el desafío personal más preocupante y 4. Enseñar en un área práctica (EF) mediante un entorno virtual, es el desafío profesional más importante. El presente estudio aporta un acercamiento a las implicaciones de la implementación de educación virtual en un área eminentemente práctica como EF y plantea cuestionamientos para investigaciones futuras.
\end{abstract}

Palabras clave: Salud, Educación Física, Educación Presencial, EducaciónVirtual, TIC.

\begin{abstract}
.
The objective of this research was to describe the challenges faced in the use of Virtual Education during the COVID-19 contingency, by 254 university professors (153 women and 102 men) from four Higher Education Institutions (HEI) in M exico, all from the area of Physical Education (PE). Analytic-synthetic, theoretical - deductive, and hermeneutic methods and quantitative and qualitative techniques through online questionnaires were used. The collected information led to the conclusion that teachers in this study: 1) Do not consider themselves capable of efficiently using virtual education; 2) Consider that virtual education, in PE, does not offer the same benefits as face-to-face education, 3) Contingency isolation is the most worrying personal challenge and 4) Teaching in a functional area (PE) through a virtual environment is the most crucial professional challenge. This study provides an approach to the implications of implementing virtual education in an eminently functional area such as $\mathrm{EF}$ and raises questions for future research.
\end{abstract}

Key words: Health, Physical Education, Classroom Education, Virtual Education, ICT.

\section{Introducción}

En 2020 el rostro de la humanidad se modificó profundamente. La presencia de la pandemia derivada de la emergencia sanitaria causada por infección del coronavirus SARS-CoV-2 (comúnmente denominada COVID-19), impactó todos los ámbitos sociales al trastocar la sal ud, la economía y las dinámicas de la vida de gran parte de la población mundial. La presencia del virushadado como resultado miles de decesos. Deacuerdo con la U niversidad de M edicina de Johns Hopkins (al día 24 de noviembre de 2020), M éxico se ubica entre los cuatro países con más decesos con 101,926; detrás

Fecha recepción: 28-09-20. Fecha de aceptación: 26-11-20 Juan Manuel Rivera-Sosa

jmrivera@ uach.mx de la India con 134,218; Brasil con 169,485 y Estados Unidos deAmérica con 259, 372 (2020).

Conforme avanza la pandemia la población está enfrentando una nueva realidad derivada de las estrategias gubernamentales, mismas que han repercutido en distintos sectores, uno de ellos la educación. En M éxico el 14 de marzo de este año, la Secretaría de Educación Pública, informó sobre las medidas de prevención para el sector educativo, las cuales acataron las disposiciones de la 0 rganización Mundial para la Salud (OMS) (Cervantes Holguín \& Gutiérrez Sandoval , 2020), 10 que implicó el cierre de los centros educativos. Esto derivó en un tránsito inmediato de la educación presencial a la educación no presencial con acercamientos alos ambientes virtuales. El profesorado de todos los niveles educativos pasó a trabajar con los estudiantes a través de diversas plataformas y tecnologías, y el alumnado 
quedó confinado en sus hogares. En pocos días se había orquestado un sistema alternativo de enseñanza y aprendizaje a distancia, sin precedentes similares, y muy estrechamente apoyado en la autogestión y la autorregulación (M uñoz M oreno \& Lluch M olins, 2020). Lo cual, trae consigo un problema propio del abrupto cambio de modalidad educativa, sin la correspondiente planeación y capacitación (Ali, 2020; Amemado, 2020; Young $\&$ Donovan, 2020). Esto supuso la pérdida de los espaciosfísicos de encuentro con otros estudiantes y con los docentes, lo cual deriva en la sensación de haber perdido la escuela, por la traslación que se ha realizado de una modalidad a la otra. De allí la importancia de analizar las condiciones en las que el sistema escolar en su totalidad-enfrentaestasituación (Díaz-Barriga, 2020), en la que la institución educativa y el hogar se convierten en uno solo. En este sentido, la teoría de la Autocomplejidad de Linville (1985), indica que los individuos necesitan contextos distintos para distintas actividades: familiares, de trabajo, diversión y educativas. A la luz de la contingencia por la COVID-19, estos contextos se han estrechado o convertido en uno a partir de la estrategia nacional de educación a distancia denominado «Aprende en Casa» (Cervantes Holguín \& Gutiérrez Sandoval, 2020), dando respuesta emergente a la situación pero con ello, también modificando la dinámica de los hogares en lo general; la casa se convierte en la escuela y en el caso de la educación básica los padres en profesores, representando con ello consecuencias psicológicas, de estrés tanto laboral como fa miliar, aún por determinar. M ientras que en los niveles medio superior y superior, el trabajo autogestionado por el estudiante y a través de medios virtuales, son los principales medios de su formación y con ello poniendo a prueba los verdaderos aprendizajes competenciales, auténticos e integrales necesarios en el futuro del profesorado de EF (López-Pastor, et al. 2016).

Lo anterior deriva en la presencia de un reto que se vive cotidianamente en los espacios educativos: los profesores reivindicando la importancia de actuar como un auténtico equipo entre profesores y padres de familia, en educación básica, y con estudiantes de manera directa en media superior y superior; liderando en ambos casos los val ores y creando climas de esfuerzo y motiva ción que sostengan la actividad formativa y académica en escuelas y universidades como auténticas comunida des de aprendizaje y particularmente, para solventar el reto de la construcción de las competencias profesionales como profesores de educación física (López-Pastor, et al. 2016). Así mismo, las familias y los estudiantes de todos los niveles educativos se han visto obligados a dotarse de tecnologías que les permitan proseguir con el programa y actividades académicas desde casa; y, singularmente, por parte de los profesionales de la educa ción para planear y seleccionar como utilizar mejor su entorno y de qué recursos disponen para mantener la continuidad del aprendizaje durante el cierre de los centros escolares (M uñoz M oreno \& Lluch Molins, 2020). En un contexto en el cual las tecnologías no van a tener un papel complementario sino determinante; y donde la docencia online se verá con otros ojos por parte de estudiantes.

El acceso a los recursos on-line a partir de herramientas digitales y la internet, sigue estando fuera del alcance de un sector amplio de la sociedad, reflejando una desigualdad y falta de acceso al uso de lasTIC, de acuerdo con los resultados de la Encuesta Nacional sobre Disponibilidad y U so de Tecnologías de la Información en los Hogares realizadaen M éxico en 2018 (Lloyd, 2020); lo cual, explica por qué para la educación básica se considera eje fundamental la transmisión de contenido por medio de laTV, con opción al uso de los medios y herramientas que sean funcionales a cada sector y comunidad escolar (para las escuelas públicas); mientras que en el nivel medio superior y superior se mantiene la educación por medio de diversas plataformas virtuales de manera emergente y bajo «modalidad híbrida», enfrentando las complicaciones correspondientes para ca pacitarse, diseñar y ejecutar una nueva docencia para la cual parece ser que no existió preparación (Silas CasiIlas \&Vázquez Rodríguez, 2020). Sin duda, el momento actual representa un cambio estructural en la educación universitaria y en la forma de impartir docencia (Torrecillas, 2020), de allí la importancia de realizar estudios que evalúen los resul tados durante esta contingencia sanitaria para la educación y la vida escolar respecto a las condiciones de trabajo de los actores de la educación y su experiencia en entornos virtuales de aprendizaje (EVA) (Cervantes Holguín \& Gutiérrez Sandoval, 2020), al tiempo que generen información sobre las estrategias adaptadas por el colectivo de los docentes universitarios en diversas universidades mexicanas, así como las formas en que se han visto afectados personalmente, y en su práctica docente, ante las nuevas condiciones que prevalecen en el entorno aca démico, social y económico.

El tránsito repentino entre la modalidad presencial y la virtual dejó vacíos en cuanto al acceso y dominio de lasTecnologías de Información y Comunicación (TIC), aspectos que se consideran como barreras tecnológicas, 
a las que se agregan la económica (no tienen acceso a Internet por no contar con recursos para hacerlo) y material (no cuentan con computadora de uso personal) (Q uintana Avello, 2020). Dichas competencias en TIC se han clasificado como básicas, de aplicación, profundización, de trabajo colaborativo, de aprendizaje permanente y éticas, y se presentan en un proceso que vadesdelanavegación básica en Internet (M aryuningsih, Hidayat, Riandi \& Rustaman, 2019), pasando por «un uso eficiente deTIC» (Infante-M oro, Gallardo-Pérez \& Infante-M oro, 2019, p. 204), resolución de problemasy análisisy tomade decisiones (Arras-Vota, Torres-Gastelú \& García-Val cárcel, 2011; Jaramillo, Cristina \& Rincón, 2011; Infante-M oro, Gallardo-Pérez \& InfanteM oro, 2019); y la interacción entre personas parallegar a un pensamiento crítico para el aprendizaje para toda la vida (Cendon, 2018; Galindo-González, et al. 2012), todo apoyado en una ética de uso deTIC que evita el plagio intelectual (O livia-Dumitrina, Casanovas \& Capdevila, 2019). Esto se habrá de subsanar conforme se van gestando las condiciones económicas, tecnológicas y de competencias en TIC de estudiantes y docentes, las cuales se refieren a «... las habilidades, capacida des y destrezas requeridas para utilizar las herramientas tecnológicas y los medios digitales» (Martzoukou, Fulton, Kostagiolas \& Lavranos, 2020).

El análisis de estrategias de enseñanza y competencias se planteó desde la percepción, definida como «... uno de los procesos cognitivos, una forma de conocer el mundo, a través de la información que aquel entrega y a partir de las experiencias de quienes perciben» (Moreno Tello, Nelly Prado \& Garcia Avendaño, 2013), es la opinión de los profesores sobre su experiencia ante los retos que enfrentan derivados de la COVID-19, así como sobre el nivel de dominio de sus competencias en TIC (Infante-M oro, Gallardo-Pérez \& Infante-M oro, 2019).

LasTIC aplicadas al contexto de la educación física $(E F)$, tienen el potencial para ser abordadas y ser medios oportunos para enfrentar el reto y los desafíos de responder a las necesidades de la educación a distancia, y particularmente desde el confinamiento por COVID19, al explorar y hacer uso de los diferentes recursos digitales aplicados a la actividad física y, en este caso, a la educación para combatir el sedentarismo desde la EF en los diferentes niveles educativos y por ende, desde los docentes del área (Hall López \& 0 chozM artínez, 2020).

En el escenario antes descrito se ubica este trabajo, el cual tiene como propósito describir, desde la pers- pectiva de los docentes Universitarios que participan en programas de formación en EF, sobre el acceso, uso y percepción respecto al manejo de lasTIC y plataformascomo recursos de enseñanza-aprendizaje en sus asignaturas on-line, así como los desafíos enfrentados ante la primera etapa de contingencia de la COVID-19, durante el semestre de enero a junio 2020 para el caso de la educación superior.

\section{Material y método}

Esta investigación de forma exploratoria, descriptiva y de campo, utilizó los métodos analítico-sintético, teórico deductivo y hermenéutico, desde el enfoque mixto que incorpora técnicas cuantitativas y cualitativas para recabar información sobre la población objeto de estudio. Es un estudio de casos, los cuales permiten comprender un fenómeno de la vida real en profundidad (Yin, 2009). Además, es un estudio fenomenológico pues presenta la experiencia sistemática de la subjetividad, de lo cotidiano, de la interpretación que otorgan, en este caso, los docentes U niversitarios del área de EF respecto a su experiencia ante la COVID-19, su percepción respecto a las TIC y a las estrategias de enseñanza-aprendizaje desarrolladas para la formación del estudiante de EF, mismas que son importantes de abordar, para descubrir las apreciaciones del profesorado (M ujica, 2020), aspecto que lo interrelaciona con la interpretación, es decir, declara el sentido o significado de sus experiencias sobre el objeto de estudio, aspecto que lo remite al enfoque hermenéutico (Stake, 1995).

\section{U niversos de estudio}

Como universos de estudio se seleccionaron cuatro facultades/ licenciaturas de EF que forman parte de cua tro Instituciones de Educación Superior (IES), que estuvieron dispuestas a participar en la investigación (Tabla 1).

El Instituto Tecnológico de Sonora (ITSO N), ofrece programas de Licenciatura en áreas de Cultura Física y el Deporte, su personal docente es de 40 profesores (2020).

La Facultad de Educación Física y del Deporte de la Universidad Autónoma de Sinaloa (UAS), trabaja con 66 profesores (2020).

La Facultad de Deportes de la U niver sidad Autónoma de Baja California (UABC), ofrece programas de licenciatura y de posgrado, su planta docente suma 86 profesores (2020)

La Facultad de Ciencias de la Cultura Física de la 
Universidad Autónoma de Chihuahua (UACh), labora con 110 profesores (2020).

\section{Muestras}

Para la obtención de las muestras se utilizó la siguiente fórmula para poblaciones finitas:

$n=$ Muestra

$$
n=\frac{z^{2} p q N}{E^{2} N+z^{2} p q}
$$

$z=1.96 \operatorname{Error}(E)=.05$

$p=$ probabilidad de éxito de la variable

$q=$ probabilidad de fracaso $(1-p)$

$\mathrm{N}=$ tamaño de la población

La selección fue probabilística, estratificada con afijación proporcional, garantizando la representatividad de la muestra en la zona de estudio, la muestra representa un $95 \%$ de confianza y se integró como se muestra en la tabla 1.

La caracterización de las muestras se resume en la tabla 2.

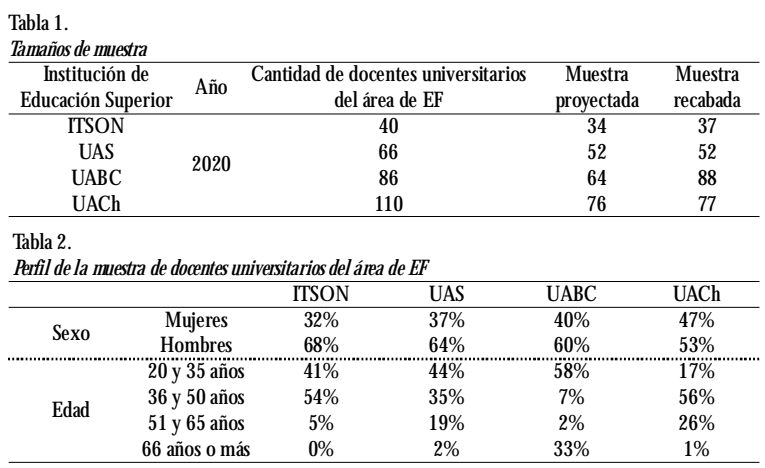

El perfil mayoritario pertenece a hombres entre 36 y 50 años de edad; las IES en su mayoría cuentan con bajo porcentaje de docentes mayoresa 65 años. LaUABC es la que registra más docentes de más de 65 años, mientras que el ITSO N no tiene docentes de más de 65 años. vivieron al pasar de la modalidad presencial a la virtual durante la pandemia.

Como punto de partida se utilizó el estudio de las habilidades en TIC en las universidades de Salamanca, España, Veracruzana y UACh (Arras-Vota, TorresGastelú \& GarcíaVal cárcel, 2011).

Cabe señalar que, para validar el instrumento, éste se envió a pares académicos y, posteriormente, se aplicó una prueba piloto, la retroalimentación vertida por estos grupos condujo a la fase final de elaboración de la encuesta que se aplicó, la cual inicia con un consentimiento informado regido por tres principios éticos.

\section{Procedimiento}

Las encuestas fueron distribuidas mediante un recopilador de datos on-line de Google Forms y la información recabada se analizó con el programa SPSS (v.22.0) a través de estadística descriptiva e inferencial. La fiabilidad (Alfa de Cronbach) de los ítems analizados, en la escala, fue de $\alpha=.831$, lo cual es aceptable.

Para el análisis de la información se utilizó el ATLAS.ti 8 donde se construyeron distintas categorías y seleccionaron las respuestas que mejor describían los resultados. Así mismo, se destacan en cursiva las ideas claves que las distinguen.

Enseguida en la Figura 1 se muestran las etapas de la investigación que se llevaron a cabo.

\section{Resultados}

Impacto de la COVID - 19 sobre docentes U niversitarios del área de EF participantes en programas educativos de EF, en su vida diaria y académica

A continuación, se presentan los resultados de 254 profesores universitarios pertenecientes a cuatro IES

\section{Instrumentos}

Como técnicas de acopio de información se utilizó una encuesta digital diseñada expresamente para este estudio, integrada por 14 ítems que se referían a la percepción de los profesores sobre el desarrollo de indicadores de competencias en TIC; mientras que las entrevistas se integraron por preguntas abiertas que indagaban las estrategias de enseñanza utiliza das y los desafíos del tránsito que

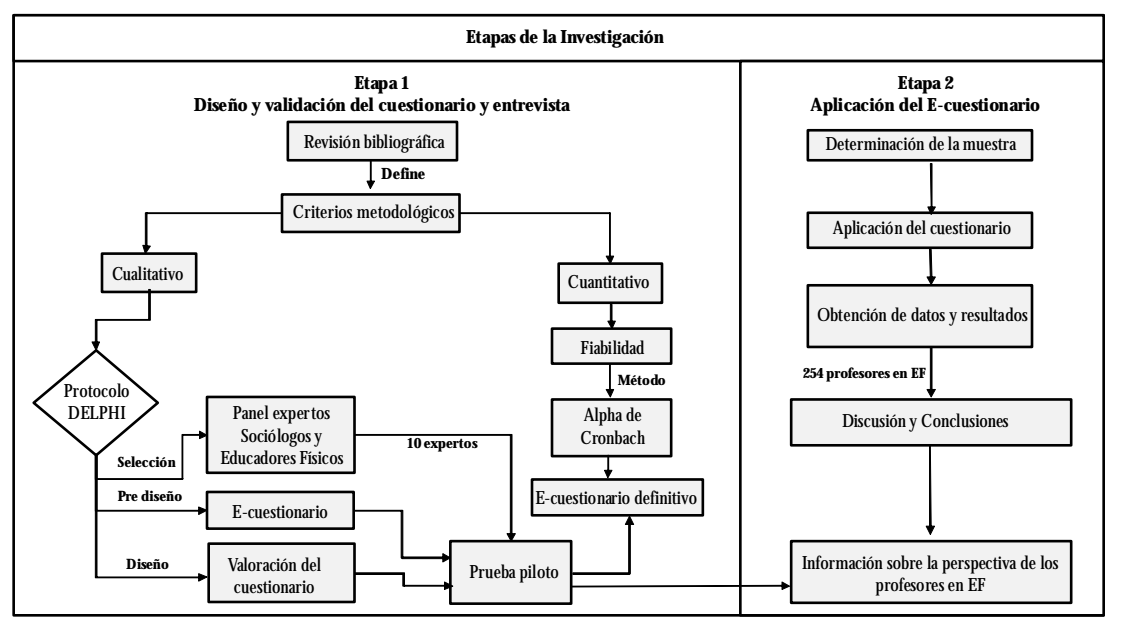

Figura 1. Procedimiento de validación del e-cuestionario y su aplicación. 
del norte de México que participan en programas de EF en donde imparten clases en los distintos niveles: 95\% (242) imparten clases en nivel Licenciatura; 20\% (52) en Maestría y 9\% (23) en Doctorado (Figura 2).

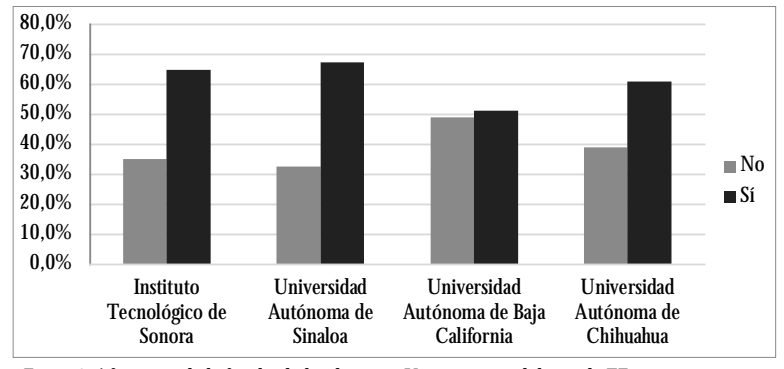

Figura 2. A fectación de la familia de los docentes Universitarios del área de EF.

Los profesores de laUAS fueron los que mayor afectación tuvieron en sus familias (67\%) seguidos por los profesores del ITSO N (65\%); en contraparte los profesores de la UABC fueron los que menos afectación tuvieron en sus familias (49\%). Con relación a losfactores que afectaron a sus familias se muestran en la Figura 3.

La figura 3 presenta la clasificación de las respuestas en 5 códigos relacionados con el ítem: afectó a la familia. Las respuestas aducen que el aislamiento ( $f=62$ ) fue lo que más afectó a las familias; seguido por la reducción de losingresos $(f=46)$ a causa del desempleo ( $f$ $=37)$; siendo el cuidado de la salud $(f=24)$ lo que menos afectó a sus familias. Algunos comentarios fueron los siguientes:

«Por el confinamiento se tuvieron que adaptar las condiciones de vida familiar, de estudios y trabajo así como las relaciones social es que esto conlleva además de las de recreación uso del tiempo libre y actividades de mantenimiento dela condición física»

«Por estar todo el tiempo en casa y no poder salir a realizar actividades al aire libre, y no tener contacto presencial con otras personas se dejaron de tener actividades deportivas y otras más»

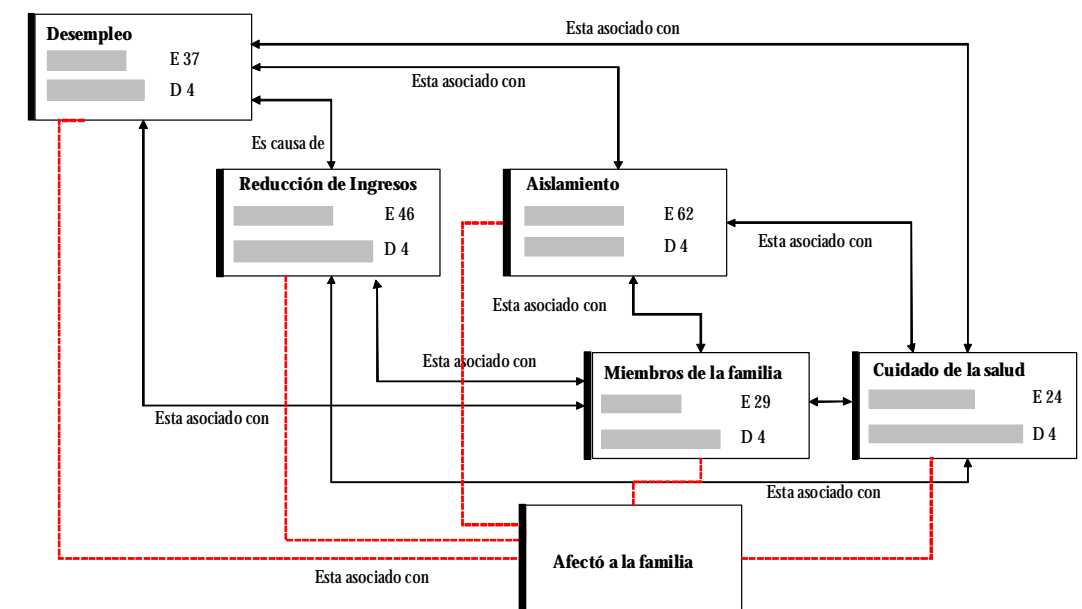

Figura 3. Red semántica de los factores por los cuales se vieron afectadas las familias de los docentes U niversitarios del área de EF.
«Por permanecer mucho tiempo en nuestras casas sin poder convivir con los demás, las noticias y rumores sobre el origen de esta enfermedad, sobre las muertes excesivas, los riesgos que hay para las personas adultas, en el descuido de las personas que nos rodean que no acatan las normas de salud»

Al realizar el análisis cualitativo de co-ocurrencias se identifica que $24 \%$ vieron afectados sus ingresos a causa del desempleo; al tiempo que $34 \%$ mencionó que la afectación de sus familias está asociada con el aislamiento.

Los profesores del ITSO N fueron los más afectados en su rol docente (95\%). Los profesores de la UABJ percibieron una menor afectación en su desempeño docente (77\%), (figura 4). Los profesores de laUABC son los que cuentan con menos recursos tecnológicos, en relación con las otras IES, como computadorae Internet, sin embargo, sintieron menos dificultades para hacer frente a su desempeño (Tabla 3).

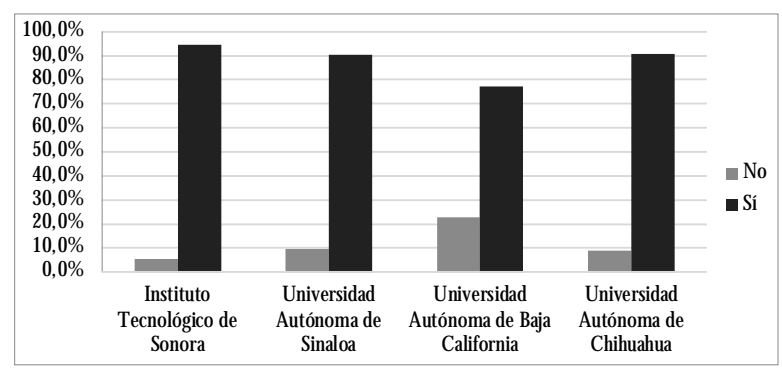

Figura 4. Afectación en el desempeño de los docentes Universitarios del área de EF.

Con relación al acceso a la Internet, los docentes universitarios ( $n=254), 93 \%(n=235)$ cuentan con red Wi-Fi; además $32 \%(n=82)$ cuentan con un Plan de Datos; así como $9 \%(n=22)$ realizan compras de Sal do (prepago).

Tabla 3.

Porcentaje de docentes universitarios del área de EF que cuentan con equipo de cómputo e internet en sus hogares

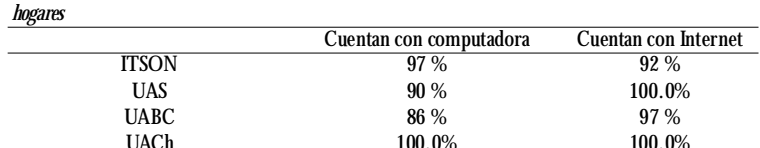
derivados del análisiscual itativo de las entrevistas con relación a la afectación que tuvieron los profesores de EF al momento de impartir sus asignaturas (Figura 5). En sus comentariosse observaque un alto porcentaje se enfocan en los impedimentos que tuvieron por el aspecto práctico de las ma terias que imparten ( $f=101$ ); seguido de la falta de experiencia 


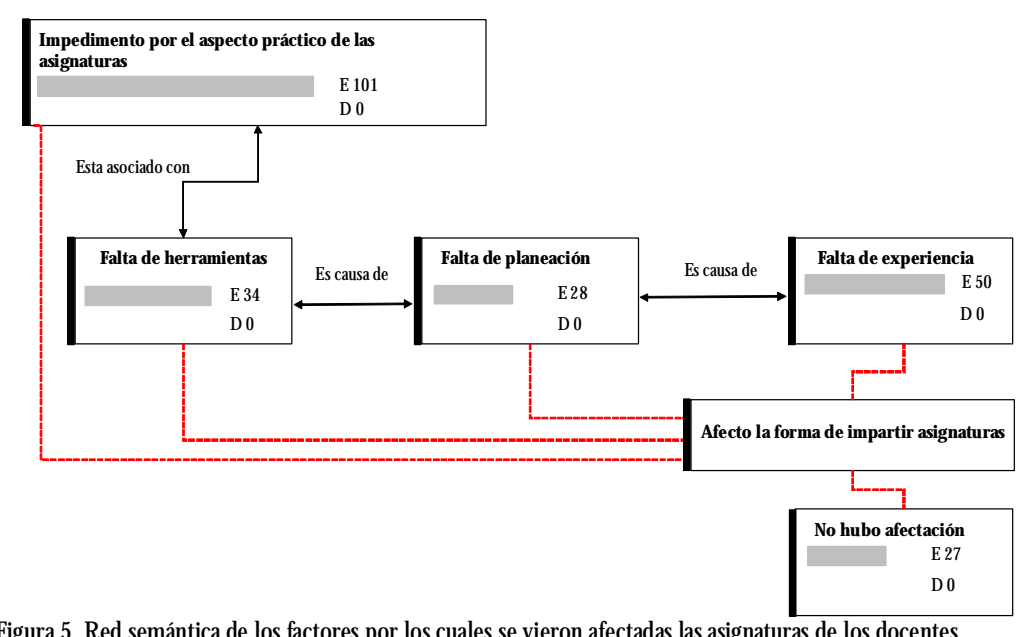

Figura 5. Red semántica de los factores por los cuales se vieron afectadas las asignaturas de los docentes universitarios del área de EF.

y herramientas para poder realizar con eficiencia la impartición de las asignaturas.

Escuchemos en voz de los participantes su experiencia:

«Al ser una clase práctica y no contar con experiencia de clases prácticas online. Encontrar formas de trabajo fue difícil»

«Se requieren estrategias nuevas, repensar las actividades apropiadas para generar en el estudiante experiencias reales de aprendizaje, ademas de estrategias de evaluacion justas, útilesy apropiadas»

«M uchas de las actividades están relacionadas con el trabajo directo con pacientes, por lo que ya no pudieron practicar en un ambiente real»

La tabla 4 muestra las dificultades que tuvieron ma yor impacto en los docentes universitarios del área de $E F$, quienes destacan que su principal reto fue acostumbrarse al trabajo desde su casa, seguido de la desinformacion y exceso de noticias basura, así como la ansiedad y estrés por la incertidumbre economica y laboral.

\begin{tabular}{|c|c|}
\hline Dificultad durante la cuarentena & Porciento \\
\hline A costumbrarse al trabajo desde su casa & $17 \%$ \\
\hline La desinfor mación y exceso de noticias basura (fake news) & $13 \%$ \\
\hline La ansiedad y estrés por la incertidumbre económica y laboral & $12 \%$ \\
\hline Cambio de planes & $11 \%$ \\
\hline O rganizar los tiempos y apegar me a ello & $11 \%$ \\
\hline Incremento en los costos de necesidades básicas & $10 \%$ \\
\hline M antener los tiempos y cronogramas de la actividad Iaboral & $9 \%$ \\
\hline No saber qué hacer en su tiempo libre & $4 \%$ \\
\hline Cierre de negocio & $3 \%$ \\
\hline Pérdida de empleo & $2 \%$ \\
\hline La convivencia 24/ 7 con pareja, familia o familiares & $2 \%$ \\
\hline Problemas familiares & $2 \%$ \\
\hline Escasez de al imentos & $2 \%$ \\
\hline
\end{tabular}

Previo a la contingencia por la COVID-19 un alto porcentaje de los docentes participantes de las IES, ca recían de experiencia en la impartición de clases a través de EVA (Figura 6), en donde el ITSO N con $51 \%$ de los docentes es el más alto, al tiempo que la U ABC con
$16 \%$ es la que menos experiencia previa contaba.

Losprofesoresdel ITSO N ma nifiestan en su mayoría un dominio intermedio (54\%) del uso de laplataformavirtual institucional; seguido de UAS, la cual en su mayoría manifiesta no saber utilizar la plataforma; la UABC con $53 \%$ de dominio intermedio y la UACh, cuyos profesores en un gran porcentaje plantean no saber utilizar laplataformacon 49\% (Figura 7).

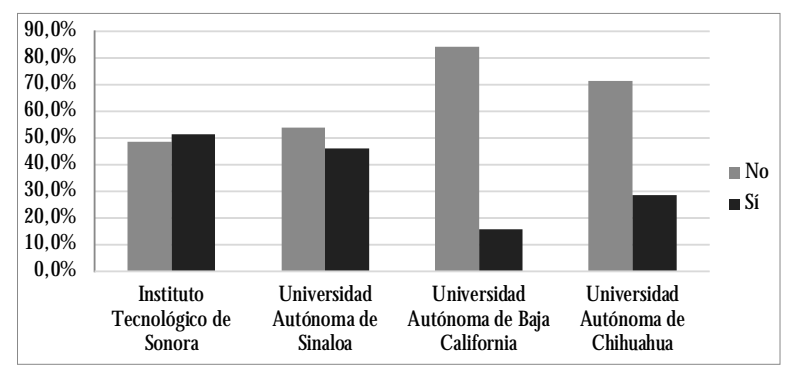

Figura 6. Experiencia previa en la impartición de clases a través de EVA.

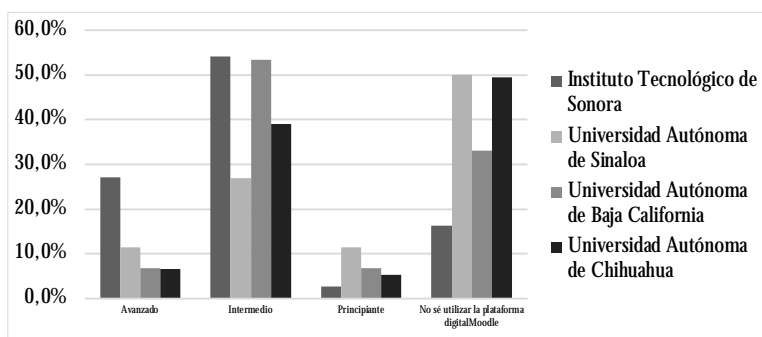

Figura 7. Percepción del nivel de experiencia en el manejo de platafor ma virtual en los docentes Universitarios del área de EF.

En tres de las IES, la mayoría de los profesores participantes aducen que impartir clases en la modalidad virtual es de su agrado, exceptuando los docentes de la UABC quienes $(57 \%$ ) manifestaron lo contrario (Figura 8).

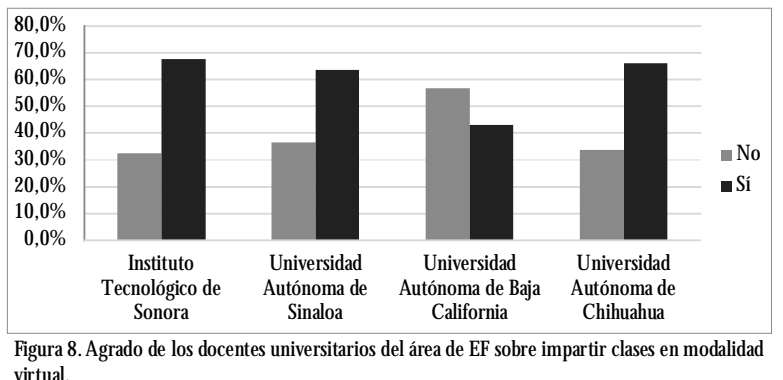
virtual.

La mayor parte de los docentes de las cuatro IES estudiadas considera que el aprendizaje es menor en la educación virtual que en la presencial (Figura 9).

Latabla5 muestralosvalores designificancia $(p<.05)$ 


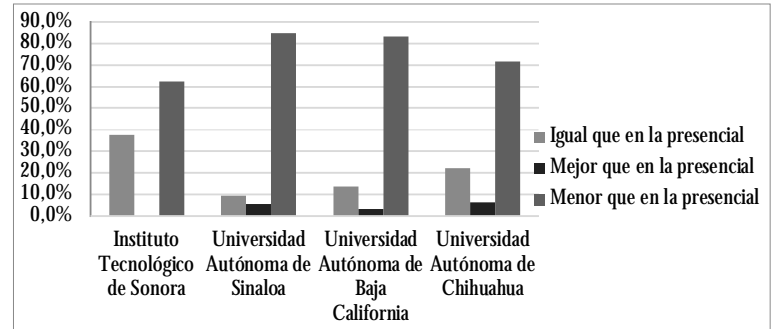

Figura 9. Como consideran los docentes Universitarios del área de EF, la eficiencia académica con la modalidad virtual.

Tabla 5.

Percepción de los docentes universitarios del área de EF participantes en el desarrollo de sus competencias en Tecnologías de Información y Comunicación

\begin{tabular}{lcccccc}
\hline & UACh ITSON & UAS & UABC & Global & $p$ \\
\hline Competencias Básicas & 3.80 & 4.32 & 3.43 & 3.65 & 3.80 & .001 \\
Competencias de Aplicación & 3.99 & 4.24 & 3.53 & 3.57 & 3.83 & .000 \\
Competencias de Profundización & 3.94 & 4.21 & 3.32 & 3.55 & 3.76 & .000 \\
Competencias deTrabajo Colaborativo & 3.84 & 4.31 & 3.82 & 3.76 & 3.93 & .058 \\
Competencias deA prendizaje para toda la vida & 4.02 & 4.37 & 3.53 & 3.43 & 3.84 & .003 \\
Competencias Éticas & 4.03 & 4.32 & 3.45 & 3.50 & 3.83 & .000 \\
\hline
\end{tabular}

La figura 10 muestra un análisis de los comentarios vertidos por los participantes sobre las plataformas digitales en TIC, dividido en 13 categorías integradoras; el uso del video - videoconferencias fue la estrategia más utilizada $(f=126)$, además del uso del Meet $(f=$ 2), Zoom ( $f=4$ ) yYouTube $(f=5)$ los cuales son plata formas que permiten establecer video conferencias. 0 tras estrategias utilizadas: W hatsApp $(f=6)$ para mantener una comunicación estrecha; Foros $(f=12)$ como medio de debate entre alumnos; Cuestionarios $(f=4)$ y Exámenes $(f=2)$; asimismo, generaron actividades que involucraban realizar investigación ( $f=12)$, uso deArtículos ( $f=2$ ) y Lecturas de distintaíndole ( $f=$ 8).

por cada una de las IES participantes. Tres instituciones muestran valores con diferencias significativas a favor del ITSO N, en las competencias básicas, aplica ción, profundización, aprendiza je para toda la vida y éticas, es en dicha institución en laque los docentesse consideran máscompetentes en TIC. En estos resultados se observa que cada una de las instituciones opina diferente con respecto a estas competencias; por otrapartelacompetencia de trabajo colaborativo la opinión de los participantes

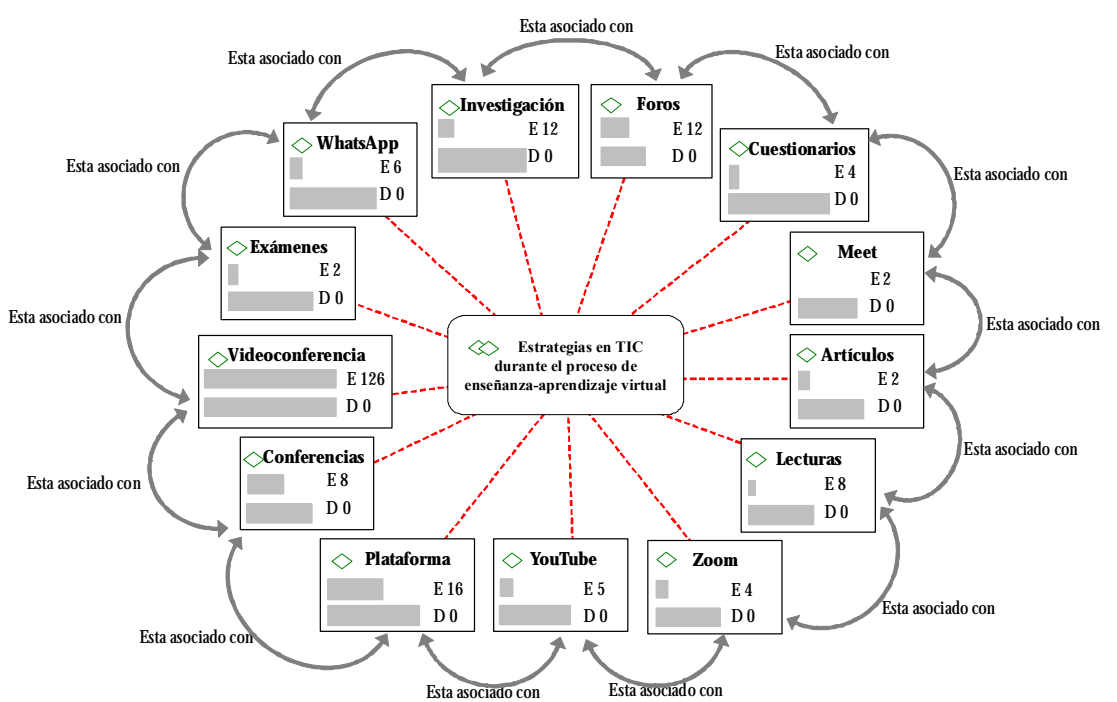

Figura 10. Estrategias en TIC que utilizaron durante el proceso de enseñanza- aprendizaje virtual. fue similar, sin diferencia significativa.

La tabla 6 presenta el tipo de contenidos curriculares de enseñanza que los profesores participantes utilizan al momento de impartir sus asignaturas prácticas. 16\% se basa en la enseñanza del proceso de aprendizaje y educación por el movimiento; seguido por $14 \%$ que trabaja con los conocimientos y ciencias relacionadas al movimiento, 14\% enseña el movimiento, mientras que $13 \%$ enseña a desarrollar el movimiento; $13 \%$ por qué se hace el movimiento, $12 \%$ qué se desarrolla con ese movimiento. $10 \%$ enseña sobre la investigación en y para el movimiento y $9 \%$ sobre los significados del movimiento.

\begin{tabular}{|c|c|}
\hline & \\
\hline Enseña sobre el proceso de aprendizaje y educación por el movimiento & $16 \%$ \\
\hline Enseña sobre los conocimientos y ciencias relacionadas al movimiento & $16 \%$ \\
\hline Ensena sobre los conocimientos y ciencias relacionadas al movimiento & $14 \%$ \\
\hline Enseña el movimiento & $14 \%$ \\
\hline Enseña a desarrollar el movimiento & $13 \%$ \\
\hline Enseña porqué se hace el movimiento & $13 \%$ \\
\hline Enseña qué se desarrolla con ese movimiento & $12 \%$ \\
\hline Enseña sobre la investigación en y para el movimiento & $10 \%$ \\
\hline Enseña sobre los significados del movimiento & $9 \%$ \\
\hline
\end{tabular}

Enseguida se muestran en voz de los participantes algunos de sus comentarios:

«Las materias que imparto son teóricas. En lo poco que se ha tenido que hacer algo práctico se han hecho videos demostrativos con instrucciones minuciosas y la manera de revisar es mediante videos del al umno siguiendo las direccioness.

«Ú nicamente explicó cómo se llevan a cabo los procedimientos de mi materia y pido a los al umnos que deben practicarlos con las personas que puedan. Por la contingencia COVID$19 »$.

«D efinitivamente que apegarme a los aspectos tecno-pedagógicos para transformar lo presencial en virtual. Resulta falsa la creencia de que virtualizar una asignatura es digitalizar documentos y subirlos a la red. Esto resulta ser un error. Se necesita aprender a construir materiales y recursos propios del entorno virtual con apoyo de recursos como: wikis, foros virtuales, formularios, hot potates, webquest, correo electrónico, chat, etc».

«Análisis de videos, creación de videos, propuesta de actividades, seguimiento de eventos deportivo-recreativos en la web, elaboración propuestas por parte de los alumnos, análisis de 
movimientos en reuniones en la web».

«Observación a través de videoconferencias, a través de videos de los alumnos, videos que manejen la técnica deportiva y situaciones tácticas, yo explicando y demostrando el movimiento o tema, etc».

\section{Discusión}

La presente investigación analiza la percepción de docentes universitarios del área de EF, sobre su quehacer académico y personal durante la contingencia de la COVID-19, en tres grandes áreas que a continuación se discuten

\section{Estrategias de enseñanza- aprendizaje desarro- lladas para sus asignaturas en e-entorno}

La videoconferencia fue la estrategia más utilizada por los participantes durante la contingencia $(f=126)$. A nivel global la tendencia de uso de videoconferencia es similar. Así lo indican informes tanto económicos (Grant, 2020; Bergen y Chang, 2020), como académicos sobre utilización de videoconferenciadurante lacontingencia (UNESCO \& IESALC, 2020). Sin embargo, trabajar en exceso con videoconferencia se reconoce más desgastante y puede ser un factor de estrés al necesitar más atención que una docencia presencial paraidentificar procesos no verbales, como expresiones faciales y tono de voz (Jiang, 2020).

En este sentido, las estrategias que permitan una interacción asincrónica con los estudiantes (wikis, foros virtuales, cuestionarios y lecturas) y un menor estrés por uso de videoconferencias, que además se encuentran disponibles en las plataformas institucionales, deberán ser una ventajaimportante (UNESCO \& IESALC, 2020). Por esto es importante resaltar que aun cuando las Instituciones participantes en este estudio cuentan con plataformas M oodle, éstas fueron utilizadas de ma nera mínima por la mayoría de los docentes (16) y apenas creciente a partir de estos periodos.

Todo lo cual indica que no se está dentro de verdaderos EVA planificados con las mejores estrategias para integrar los e-entornos, solo se responde a una emergencia (UNESCO \& IESALC, 2020) cuyasimplicaciones formativas están aún por descubrirse (Amemado, 2020; Giridharan, 2020; Young \& Donovan, 2020).

\section{Su percepción con respecto a las Tecnologías de Información y Comunicación (TIC)}

Lapercepción docente con respecto a lasTIC se presenta en este estudio en dos vertientes:
La personal: los docentes de las cuatro IES, tienen, en su mayoría, una percepción positiva sobre el desa rrollo de sus competencias personales en cuanto a uso deTIC, indicando que impartir clases en la modalidad virtual les agrada y que se consideran aptos para ello. Además, indican que no contar con las herramientas de TIC, no sería un factor decisivo para continuar con sus labores docentes en esta área.

La profesional-docente: se indica lo contrario ya que en su mayoría consideran que el aprendizaje sería menor en educación virtual, y al menos la mitad de los docentes (en el caso de la U ABC) no se sienten plenamente capacitados para trabajar cursos online, lo cual concuerda con investigaciones que presentan una importante falta de capa citación docente sobre uso de TIC, especialmente en IES (Groff, 2013; Jacobsen, Brown \& Lambert, 2013; Sun \& Chen, 2016; Hodges, et al. 2020). Q ueda por explorar, hasta donde esta percepción es construida por el área profesional en que participan como docentes universitarios.

Desde la perspectiva de M ercader y Gauirin (2020) existen barreras tanto personal es (percepción sobre uso de TIC) como institucionales y contextuales (falta de capacitación) que inciden en una aplicación eficiente de tecnologías digitales en educación. Los docentes participantes en el estudio se perciben capaces, pero no ca pacitados lo que incide en su desempeño docente.

\section{Los desafíos enfrentados ante la contingencia de la COVID - 19}

Cuatro desafíos sobresalen en opinión de los docentes, durante la contingencia: trabajo, familia-aislamiento social, uso del tiempo libre y cuidado de la salud.

\section{Trabajo}

La mayoría de los docentes indicaron que su trabajo docente se vio afectado por la contingencia, lo cual es congruente con la tendencia mundial (ONU, 2020; UNESCO \& IESALC, 2020). El factor más citado fue el impedimento por el aspecto práctico de las asignaturas ( $f=101$ ) indicando la preocupación ante la imposibilidad de trabajar en un ambiente real. Esto está muy relacionado con la percepción característica en EF de tra bajar de manera presencial, al igual que aspectos prácticos y en ambientes reales (Fitzpatrick \& Pope, 2005). Es de resaltar que este factor sea aún más importante que poca experiencia de trabajo online o no contar con las herra mientas adecuadas o capacitación institucional, factores a los que no le otorgan tanta importancia. ¿Podrá esto deberse a que, frente a una situación extraordinaria los 
docentes consideren que estos factores no serán toma dos con la misma importancia como la disposición y adaptación haciael trabajo online?D eacuerdo con Cleary \& Zimmerman (2004), todo cambio de un ambiente habitual (salón de clases) a un nuevo ambiente (virtual) afectará aun a los participantes más exitosos, quienes deberán estar dispuestos, adaptarse y activar nuevas estrategias de autorregulación; a la vista de la urgencia actual por el cambio de modelo, es poco probable que dichas adaptaciones ocurran de manera inmediata, aún y cuando en lo profesional ya se advierte el reconocimiento de que estos escenarios son oportunos para el ámbito de la EF (Mondaca-Fernández, 2019; Hall \& 0 choa, 2020). Así mismo, se reconoce la necesidad de mantenerse cerca de las prácticas simuladas en la realidad y de exposición gradual para provocar el aprendiza je docente necesario y una intervención didáctica más asertiva (Alonso, et al. 2016).

Sin embargo, se requiere que los directivos de las IES realicen acuerdos con los docentes para que el tipo de enseñanza sobre el movimiento sea más integral, con un enfoque similar y contextualizado a los nuevos escenarios de salud, sociales, económicos y profesiona les que hoy se reconocen como retos relacionados al área de EF (López-Pastor, et al. 2016). Es necesario potenciar y promover la importancia tanto de la EF como de los factores inherentes a su ejercicio, aprendizajes para sus destinatarios, los escolares de la educa ción básica (León, et al. 2020; Rivera-Sosa, et al. 2021), para ello, se converge que se debe estudiar en lo sucesivo tanto los estil os de enseñanza de los docentes, como de aprendizaje de los estudiantes (BahamondesA cevedo, et al. 2021), pero ahora considerando el desarrollo de su formación a través y por estos medios online.

\section{Familia}

La familia también se vio afectada a través del aisla miento social en casa ( $f=62$ ) para al menos casi la mitad, los docentes en sus respuestas plantearon cambios en las condiciones de vida familiar y en sus dinámicas vinculadas con el aislamiento social, mismo que se menciona como uno de los factores importantes que están impactando negativamente a la población en general y alas IES durante esta pandemia(O MS, 2020; UNESCO \& IESALC, 2020). Además, con base en laTeoría de la Auto-complejidad (Linville, 1985) los individuos necesitan realizar sus diversos roles sociales como educación, ocio, y familia, cada uno en su propio contexto. M ientras tanto debido a la pandemia la mayoría de estos procesos sociales están sucediendo en un mismo es- pacio, el hogar, con un alza en resultados negativos para la salud mental y física (J iang, 2020).

\section{Uso de tiempo libre}

Un factor con gran atención durante la pandemia: ¿Q ué hacer con el tiempo libre que se tendrá en casa? En todo el mundo se han mostrado signos tanto de preocupación (Hammami, et al. 2020) como de optimismo (Hudson \& Sprow, 2020) sobre las posibilidades que la pandemia brindará sobre el uso del tiempo libre. En el área de EF se indica que la dupla docente de EF-padres de familia son una conjunción y apoyo importante para poder utilizar el tiempo libre positivamente (M CD avid, et al. 2012), especialmente durante la actual contingencia, lo cual requiere del uso e implementación de estra tegias bajo los recursos y herramientas de lasTIC como medios no alternativos, sino básicos para cubrir las necesidades emergentes por la pandemia.

Por esto, es de resaltar que $11 \%$ los docentes universitarios de este estudio- indican no saber qué hacer con su tiempo libre. ilmplica esto que los docentes en el estudio realmente no tienen las habilidades para saber utilizar positivamente su tiempo libre? ¿es una percepción propia del docente y ajena a su preparación profesional? 0 ¿corresponde a docentes con perfil profesional no del área de EF y por tanto explicativo de esta discrepancia? Estos casos abren la puerta a investigaciones para clarificar estos posibles escenarios.

\section{El cuidado de la salud}

El cuidado de la salud es el mayor desafío actual a nivel mundial (Nadu, 2020; UNESCO \& IESALC, 2020). Sin embargo, para los docentes en este estudio es el factor menos desafiante en cuanto a la afectación a sus familias. El ser parte del sector educativo, el cual para la O NU (2020) está catal ogado como uno de los de más bajo riesgo económico, y además estar bajo contrato en las IES, asegura servicio médico, implicando la cobertura del factor tan importante de cuidado de la salud. Esto representa la base para trabajar de manera positiva, con seguridad y distinta a otras poblaciones en al to riesgo durante la pandemia como el área médical hospitalaria.

\section{Conclusiones}

En el presente estudio se infiere que los docentes participantes de las cuatro IES presentadas, responden ante la contingencia por COVID-19 de una manera similar al resto de los docentes del mundo: utilizan estra- 
tegias a través de lasTICs y Plataformas proporcionadas por sus instituciones sin posibilidad de adaptarse a ellas mediante un adecuado proceso; perciben la educación online como un medio idóneo, pero que al aplicarse con premura genera dudas sobre su efectividad; $y$, además, enfrentan desafíos comunes durantelacontingencia, tanto en familia como en el trabajo. Sin embargo, también cuentan con factores a favor, como seguridad económica y de salud, que pueden ayudarlos a adaptarse a esta contingencia de una mejor manera y con oportunidad para desempeñarse en su quehacer educativo.

En cuanto a esta migración forzada a los ambientes online podemos concluir que las IES en esteestudio cuentan con programas de diplomados y cursos para impartición de clases en EVA y su propia plataforma online, lo cual debe verse como una ventaja. Con base en esto se recomienda trabajar de manera conjunta para revisar las estrategias y actividades online de cada IES que puedan apoyar a las demás a obtener los mejores resultados en el mínimo tiempo.

Finalmente, el cambio a educación online que esta mos viviendo no se presentó por resultado de una planificación pertinente. Fue la contingencia por COVID-19 lo que obligó a llevar la educación universitaria a medios virtuales, con premura y sin la capacitación y tecnología adecuadas para ello. Por lo cual, no estamos viviendo unaeducación online formal y losproblemas aquí indicados no parecen representar a un EVA planeado de manera eficiente. Los problemas como falta de capacitación en TIC y en uso de sistemas M oodle son factibles de cubrir en el corto y mediano plazo. Son los factores de estrategias educativas, manejo de problemas familiares y percepción sobre los resultados académicos del uso de EVA, los que se deben trabajar de manera imperiosa, eficiente y permanente para obtener una educación online de calidad; especialmente si esta contingencia finaliza antes de haber logrado ese objetivo.

\section{Agradecimientos}

Nuestro agradecimiento por el apoyo para realizar esta investigación a: Dr. Luis Roberto Monreal O rtiz, UAS; Dr. Iván de JesúsToledo Domínguez, ITSO N ; Dr. Javier Arturo Hall López, Dr. Iván Rentería, Dra. Gabriela Valles Verdugo y M.C. Luis Mario Gómez Miranda, UABC.

\section{Referencias}

Ali, W. (2020). O nline and Remote Learning in Higher
Eduction Institutes: A N ecessity in light of COVID-19 Pandemic. Higher Education Studies, 10(3), 16. https:/ / doi.org/ 10.5539/ hes.v10n3p16

Alonso, M. C., Gómez-Alonso, M. T., Pérez-Pueyo, Á. \& Gutiérrez-García, C. (2016). Errores en la intervención didáctica de profesores de educación física en formación: perspectiva de su compañeros en sesiones simuladas Retos, (29), 229-235. https/ / doi.org/ 10.47197/ retos. v0i29.40966

Amemado, D. (2020). COVID-19: An Unexpected and Unusual D river to O nline Education. International H igher Education, 102(Special Issue 2020), 12-14. https:/ / www. internationalhighereducation. net/ api-v1/ article/ !/ action/ getPdfO fArticle/ articlelD/ 2922/ productID/ 29/ filename/ article-id-2922. pdf

Arras-Vota, A. D. , Torres-Gastelú, C. A. \& García-Valcárcel, A. (2011). Student's perceptions about their competencies in Information and Communication Technologies (ICTs). Revista Latina de Comunicación Social (\# 66), 1-26. http:/ / dx. doi.org/ 10.4185/ rics-66-2011927-130-152-en

Bahamondes Acevedo, V., Flores Ferro, E., Maureira Cid, F., VargasVitoria, R., Gavotto Nogales, O., Véliz Véliz, C. \& Aravena Garrido, C. (2021). Percepción de los estudiantees de Educación Física sobre el desempeño del profesorado. Retos, (40), 180-185. https:/ / doi.org/ 10.47197/ retos. v1i40.82160

Bergen, M . \& Chang, E. (2020,A pril 13). Technology: YouTube Sees $75 \%$ Jump in NewsViews on Thirst forVirus U pdates. Abril 2020. https:/ / www. bloomberg.com/ news/ articles/ 2020-04-13/ youtube-sees-75-jump-in-newsviews-on-thirst-for-virus-updates

Cendon, E. (2018). Lifelong learning at universities: future perspectives for teaching and learning Journal of $\mathrm{N}$ ew Approaches in Educational Research , 7(2), 81-87. https:/ / doi.org/10.7821/ nær.2018.7.320

Cervantes Holguín, E. \& Gutiérrez Sandoval , P. (2020). Resistir la Covid-19. Intersecciones en la Educación de Ciudad Juárez, M éxico. Revista Internacional de Educación para la Jusicia Social, 9(3), 1-23. https:/ / revistas. uam.es/ riejs/ article/ view/ riejs2020_9_3_001

Cleary, J. T. \& Zimmerman, J. B. (2004). Self-regulation empowerment program: A school- based program to enhance self-regulated and self-motivated cycles of student learning. Psychology in the Schools, 41(5), 537550. https:/ / psycnet. apa org/ record/ 2004-13735-005 Díaz-Barriga, A. (2020). La escuela ausente, la necesidad de replantear su significado. In IISUE, Educación y pandemia, una visión académica (pp. 19 - 29). México: Instituto de Investigaciones sobre la U niversidad y la Educación de 
la UNAM. http:/ / 132.248.192.241:8080/ xmlui/ bitstream/ handle/ IISUE_UNAM / 535/ DiazBarrigaA_2020_La_escuela_ausente . . pdf?sequence $=1 \&$ isAllowed $=y$

Fitzpatrick, K. \& Pope, C. (2005). Is physical education relevant? Interpersonal skills, values and hybridity. ACH PER Healthy Lifestyles Journal, 52(3), 24-29. http:/ / citeseerx. ist.psu.edu/ view doc/ download?doi=10.1.1.876.1595\&rep=rep1\&type=pdf

Galindo-González, R. M., Galindo Gonzélez, L., Martínez de la Cruz, N., Ley Fuentes, M. G., RuizAguirre, E. I. \& Valenzuela González, E. (2012). Acercamiento epistemológico a la teoría del aprendizaje colaborativo. Apertura, 4(2), 159-159. https:/ / www. redalyc. org/ pdf/ 688/ 68829135010.pdf

Giridharan, B. (2020) Engaging with Students and Faculties O nline in the Era of the CoronaVirus Pandemic: A Higher Education Perspective ( Prepublicación online) Horizon J. H um. \& Soc. Sci. 2(S), 103-110. https:/ / w w w. researchgate.net/ publication / 342210851_Engaging with_Students_and Faculties_Online in the_Era_of the Corona_Virus_Pandemic_ $\bar{A}$ Higher_Education_Perspective

Grant, N. (2020, June 2). Technology: ZoomTransforms H ype Into Huge Jump in Sales, Customers. https:/ / www. bloomberg.com/ news/ articles/ 2020-06-02/ zoom-transforms-hype-into-huge-jump-in-salescustomers

Groff, J. (2013). Technology-rich innovative learning environments. OCED CERI Innovative Learning Environment project. 1-30. http:/ / www. oecd.org/ ed u cation / ceri / T echnolog y Rich\%20lnnovative\%20Learning\%20Environments\%20 by\%20J ennifer\%20Groff.pdf

Hall López, J. A. \& 0 choa-M atínez, P.Y. (2020). Enseñanza virtual en educación física en primaria en México y la pandemia por COVID-19. Revista Ciencias de la Actividad Físca, 21(2), 1-7. https/ / doi.org/ 10.29035/ rcaf.21.2.4 Hammami, A., Harrabi, B., M ohr, M.\& Krustrup, P. (2020). Physical activity and coronavirus disease 2019 (COVID19): specific recommendations for home-based physical training Managing Sport and Leisure, 0(0), 1-6. https:/ / doi.org/ 10.1080/ 23750472.2020.1757494

Hodges, C., Moore, S., Lockee, B., Trust, T. \& Bond, A. (2020). The Difference Between Emergency Remote Teaching and 0 nline Learning Educase. March 27. Retrieved from https:/ / er. educause edu/ articles/ 2020/3/ the-difference-between-emergency-remoteteaching-and-online-learning

Infante-Moro, J. C., Gallardo-Pérez, J. \& Infante-Moro, A.
(2019). The importance of ICTs fos Students as a Competence for their Future Professional Performance: The Case of the Faculty of Business Studies and Tourism of th University of HuelvaAlfonso. Journal of New Approaches in Educational Research, 8(2), 201-213. https: / / dialnet.unirioja.es/ servlet/ articulo?codigo $=7000282$

InstitutoTecnologico de Sonora (2020, 07 17). Oferta Acadé mica. Retrieved from https: / / www. itson.mx/ oferta/ Paginas/ ofertaacademica. aspx

Jacobsen, M., Brown, B. \& Lambert. D. (2013). Technologyenhanced learning environments in higher education: $A$ review of the literaure. Werklund School of Education, University of Calgary. Retrieved from http:/ / hdl. handle.net/ 1880/ 52244

Jaramillo, P., Cristina, H. \& Rincón, Y. (2011). ¿Cómo ma nejan información los estudiantes de educacion superior? El caso de la U niversidad de La Sabana, Colombia. Información, cultura y sociedad, 117-143. https:// www. redalyc. org/ articulo. oa?id=263030844007

Jiang, M. (2020,A pril 22). Video chat ishelping us stay employed and connected. But what makes it $s 0$ tiring - and how can we reduce 'Zoom fatigue'? BBC. https:/ / www. bbc.com/ worklife/ article/ 20200421-why-z00m-video-chats-areso-exhausting

Johns Hopkins University and Medicine. (2020, 07 02). Coronavirus Resource Center. Retrieved from https:/ / coronavirus.jhu. edu/ map. html

Linville, P.W. (1985), Self-complexity and affectiveextremity: Don't put all of your eggs in one cognitive basket. Social Cognition, 3, 94-120. https:/ / doi. org/ 10.1521/ soco.1985.3.1.94

Lloyd, M. (2020). Desigualdadeseducativasy labrechadigital en tiempos de COVID-19. Educación y pandemia: una visón académica, 115-121. http:/ / 132.248.192.241:8080/ xmlui/ bitstream/ handle/ IISUE UNAM / 546/ LloydM_2020_Desigualdades_educativas. pdf?se quence $=\overline{1}$ \&isAllowed=y

López Pastor, V. M., Pérez Brunicardi, D., Manrique Arribas, J. C. \& MonjasAguado, R. (2016). Los retos de la Educación Física en el Siglo XXI. Retos, (29), 182-187. https: / / doi.org/ 10.47197/ retos. v0i29.42552

Martzoukou, K., Fulton, C., Kostagiolas, P. \& Lavranos, C. (2020). A study of higher education students selfperceived digital competences for learning and everyday life online participation. Journal of Documentation, 76(6), 1-51. https:// www. emerald. com/ insight/ content/ doi/ 10.1108/ JD-03-2020-0041/ full/ html

Maryuningsih, Y., Hidayat, T., Riandi, R. \& Rustaman, N.Y. (2019). Profile of information and communication 
technologies (ICT) skills of prospective teachers. Journal of Physics: Conference Series, 1521, 1-8. https:/ / iopscience.iop.org/ article/ 10.1088/ 1742-6596/ 1521/ 4/ 042009/ pdf

McDavid, L., Cox, A. E. \& Amorose, A. J. (2012). The relative roles of physical education teachers and parents in adolescents' leisure-time physical activity motivation and behavior. Psychology of Sport and Exercise, 13(2), 99107. https:// doi.org/10.1016/ j.psychsport.2011.10.003

Mercader, C. \& Gairín, J. (2020). U niversity teachers' perception of barriers to the use of digital technologies: the importance of the academic discipline. International Journal of EducationalTechnology in Higher Education, 17(1). https:/ / doi.org/ 10.1186/ s41239-020-0182-x

Mondaca-Fernandez, F. (2019). Technology Enhanced Learning Environments within Physical Education Teacher Education:A pplication of Self-Regulated learning and Self-Determination Theory. Doctor of Philosophy thesis, School of Education, University of Wollongong, 2019. https:/ / ro. uow. edu. au/ theses1/ 560/

Moreno Tello, M. A., Nelly Prado, E. \& GarciaAvendaño, D. J. (2013). Percepción delosestudiantes deenfermeria sobre el ambiente de aprendizaje durante sus practicas clinicas. Cuidarte, 4(1), 444-449. http:// ww w. sciel 0. org. co/ scielo. php?pid=\$2216$09732013000100003 \&$ script=sci_abstract\&tlng=es

Mujica, F. (2020). El término Educación Física en la postmodernidad: contribución de algunas perspectivas fenomenológicas. Retos, (38), 795-801. https:/ / doi.org/ 10.47197/ retos. v38i38.73011

MuñozM oreno, J. L. \& LluchM olins, L. (2020). Educacióny Covid-19: Colaboración de las Familias y Tareas Escola res. Revista Internacional de Educación para la Jusicia Social, 9(3), 1-17. https: / / revistas. uam.es/ riejs/ article/ view/ 12182

Nadu,T. (2020). ILO Sectoral Brief. 2019 (April).

Olivia-Dumitrina, N., Casanovas, M. \& Capdevila,Y. (2019). Academic W riting and the Internet: Cyber-Plagiarism amongst University Students Journal of N ew Approaches in Educational Research, 8(2), 112-125. https:/ / doi. org/ 10.7821/ nær.2019.7.407

León, M. P., Prieto-Ayuso, A. \& Gil-Madrona, P. (2020). Hábitos y motivos de ejercicio físico en estudiantes universitarios y su relación con el valor otorgado a laEducación Física. Retos, (37), 78-84. https:/ / doi.org/ 10.47197/ retos. v37i37.70454

OMS. (2020). M ental healthand psychosocial considerations during the COVID-19 outbreak. M arch 2020. WHO referencenumber:W HO / 2019-nCoV/ M entalHealth/ 2020.1 https:// www. who.int/ docs/ default-source/ coronaviruse/ mental-health-considerations.pdf

ONU (2020). Policy Bruief:TheWorld ofWork and COVID-19 JUNE 2020 . June.

Quintana Avello, I. (2020). Consecuencias del Cierre de Escuelas por el Covid-19 en las Desigualdades Educativas. Revista Internacional de Educación para la Jusicia Social, 9(3), 1-11. https:/ / revistas.uam.es/ riejs/ issue/ view/ riès2020_9_3

Rivera Sosa, J. M. ., ArrasVota, A. M., Tarango, J., M endoza Meraz, G. \& LópezAlonzo, S. J. (2021). Educación física: Referentes y nociones conceptuales del personal docenteEnEducación Primaria Retos, (39), 298-305. https:/ / doi.org/ 10.47197/ retos. V0i39.58114

Silas Casillas, J. C. \& Sylvia, V. R. (2020). El docente universitario frente a las tensiones que le plantea la pandemia. N ueva Época, 89-120. https: / / www. redalyc.org/ jatsRepo/ 270/ 27063237022/ html/ index.html

Stake, R. E. (1995). TheArt of Case Study Research. London: Thousand Oaks: SAGE Publications.

Sun, A. \& Chen, X. (2016). O nlineeduction and itseffective practice: A research review. Journal of Information Technology Education Research, 15, 157-190. Retrieved from http:/ / ww w. jite. org/ documents/ Vol15/ JITEv15ResearchP157-190Sun2138.pdf

Torrecillas, C. (2020). El reto de la docencia online para las universidades públicas españolas ante la pandemia del Covid-19. ICEl Papers, 16, 1-4. https: / / www.ucm.es/ icei/ file/ iceipapercovid16

UNESCO \& IESALC. (2020). COVID-19 and higher education: Today and tomorrow. Impact analysis, policy responses and recommendations. lesalc, 1-46. https: / / bit. ly/ 34TO Svu

Universidad Autónoma de Chihuahua. (2020, 01 1). Estudios. Retrieved from https: / / uach. mx/

Universidad Autónoma de Chihuahua. (2020, 07 31). Facultad de Ciencias de la Cultura Física. Retrieved from http:/ / www.fccf.uach. mx/

Universidad Autónoma de Chihuahua (2018, 09 20). H istoria. Retrieved from https: / / uach.mx/ acerca/ historia/ Universidad Autónoma de Baja California (2020, 07 17). Numeralia Institucional. Retrieved from http:/ / www. uabc. mx/ planeacion/ numeralial

Universidad Autónoma de Sinaloa. (2020, 07 17). Inicio. Retrieved from https:/ / www.uas.edu. $\mathrm{mx}$ / index.php Yin, R. (2009). CaseStudy Research. D esign and M ethods (4 ed.). London, UK:Thousand O aks: Sage Publications.

Young, J. \& Donovan,W. (2020). Shifting to 0 nline Learning in the COVID-19 Spring Pioner Institute: Public Policy Research, April, 1-6. https:/ / files eric. ed. gov/ fulltext/ ED604252.pdf 